Conclusions: The prevalence of autoantibodies is similar in HOA patients and healthy controls. Moreover, these autoantibodies are not associated with erosive disease, structural damage or inflammation in HOA patients, indicating that another mechanism is driving erosive disease.

Acknowledgements: We thank J.C. Kwekkeboom for her help in collecting the samples, C. Kromme for his help with designing the data file and W. Damman for her help in the scoring of the radiographs.

Disclosure of Interest: M. van Delft: None declared, S. Van Beest: None declared, M. Kloppenburg: None declared, L. Trouw Consultant for: Listed as inventor in a patent application regarding the detection of anti-CarP antibodies for rheumatoid arthritis, A. loan-Facsinay: None declared

DOI: 10.1136/annrheumdis-2018-eular.2584

\section{SAT0580 ASSOCIATION OF CHILDHOOD AND ADULTHOOD ADIPOSITY MEASURES WITH KNEE CARTILAGE THICKNESS, CARTILAGE VOLUME AND BONE AREA IN YOUNG ADULTS}

T. Meng ${ }^{1}$, A. Venn ${ }^{1}$, F. Eckstein ${ }^{2,3}$, W. Wirth ${ }^{2,3}$, F. Cicuttini ${ }^{4}$, L. March ${ }^{5}$, T. Dwyer ${ }^{1,6}$ M. Cross ${ }^{5}$, L.L. Laslett ${ }^{1}$, G. Jones ${ }^{1}$, C. Ding ${ }^{1,4,7}$, B. Antony ${ }^{1} .{ }^{1}$ Menzies Institute for Medical Research, Hobart, Australia; ${ }^{2}$ Institute of Anatomy, Paracelsus Medical University Salzburg and Nuremberg, Salzburg, Austria; ${ }^{3}$ Chondrometrics GmbH, Ainring, Germany, ${ }^{4}$ Department of Epidemiology and Preventive Medicine, Monash University, Melbourne; ${ }^{5}$ Institute of Bone and Joint Research, University of Sydney, Sydney, Australia; ${ }^{6}$ The George Institute for Global Health, Nuffield Department of Obstetrics and Gynaecology, University of Oxford, Oxford, UK; ${ }^{7}$ Clinical Research Centre, Zhujiang Hospital, Southern Medical University, Guangzhou, China

Background: Adiposity is associated with increased risk of knee osteoarthritis $(\mathrm{OA})$; cartilage thickness, cartilage volume and subchondral bone area are established biomarkers in knee OA. However, there are no studies describing the effects of adiposity during early life on knee cartilage and bone morphology in adulthood.

Objectives: To describe the longitudinal associations between adiposity measures in childhood and adulthood and knee cartilage thickness, cartilage volume and subchondral bone area in young adults.

Methods: 186 participants from the Australian Schools Health and Fitness Survey of 1985 (aged 7-15 years) were followed up 25 years later (aged 31-40 years). Childhood measures (weight, height, waist circumference and hip circumference) were collected in 1985, and corresponding adulthood measures were collected during 2004-2006. Body mass index (BMI) and waist-hip ratio (WHR) were calculated. Participants underwent knee magnetic resonance imaging (MRI) during 2008-2010, and cartilage thickness, cartilage volume and subchondral bone area were measured using a quantitative approach (Chondrometrics 3.0, Germany). Multivariable linear regressions were used to examine the above associations.

Results: Among 186 participants (48.4\% females), 7.6\% were overweight in childhood, and $42.1 \%$ in adulthood. There were no significant associations between childhood adiposity measures and adulthood knee cartilage and bone morphological measures; the same applied to adulthood BMI and overweight. However, adulthood WHR was negatively associated with cartilage thickness in the patella ( $\beta-0.009 \mathrm{~mm} / 0.01$ unit, $95 \%$ confidence interval $(\mathrm{Cl})-0.019$ to 0.001 , $\mathrm{p}=0.065)$, the medial femorotibial compartment (MFTC) $(-0.011,-0.022$ to 0.000 $\mathrm{p}=0.048)$ and the lateral femorotibial compartment (LFTC) $(-0.013,-0.025$ to $-0.002, p=0.027)$, with cartilage volume in the patella $\left(\beta-21.85 \mathrm{~mm}^{3} / 0.01\right.$ unit, $95 \% \mathrm{Cl}-37.77$ to $-5.93, \mathrm{p}=0.007)$, the MFTC $(-14.17,-29.53$ to $1.19, \mathrm{p}=0.070)$ and the LFTC $(-23.87,-42.18$ to $-5.57, \mathrm{p}=0.011)$, and with subchondral bone area in the patella $\left(\beta-4.69 \mathrm{~mm}^{2} / 0.01\right.$ unit, $95 \% \mathrm{Cl}-8.26$ to $\left.-1.12, \mathrm{p}=0.010\right)$ and the LFTC $(-4.76,-9.47$ to $-0.06, p=0.047)$, but not the MFTC.

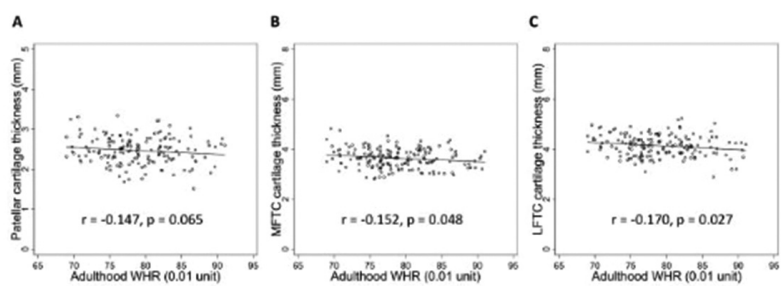

Abstract SAT0580 - Figure 1. Scatter plots and linear regression lines for associations between adulthood WHR and knee cartilage thickness (A: patella; B: MFTC; C: LFTC).

Linear regression lines are from models adjusted for adulthood age, duration of follow-up, gender and adulthood knee injury. WHR, waist-hip ratio; MFTC, medial femorotibial compartment; LFTC, lateral femorotibial compartment.
Conclusions: Childhood adiposity measures did not predict adulthood knee cartilage and bone morphological measures. However, adulthood WHR, but not BM or overweight status, was negatively associated with cartilage thickness, cartilage volume and subchondral bone area, suggesting central obesity may affect knee structures in young adults.

Disclosure of Interest: None declared

DOI: 10.1136/annrheumdis-2018-eular.2649

\section{SAT0581 PREDICTIVE FACTORS OF RESPONSE TO A SINGLE INJECTION OF MANNITOL-MODIFIED CROSS-LINKED HYALURONIC ACID (HANOX-M-XL) IN PATIENTS WITH TRAPEZIOMETACARPAL OSTEOARTHRITIS. RESULTS OF A MULTICENTRE PROSPECTIVE OPEN-LABEL PILOT STUDY (INSTINCT TRIAL)}

J. Dauvissat ${ }^{1}$, T. Conrozier $^{1}$, H. Lellouche ${ }^{2}$, B. Maillet ${ }^{3}$, C. Rizzo ${ }^{4}$, V. Travers ${ }^{5}$, V. locquet ${ }^{6}$, S. Mellac-Ducamp ${ }^{7} .{ }^{1}$ Rheumatology, Nord Franche Comté Hospital, Trevenans; ${ }^{2}$ Rheumatology, Institut Nollet, Paris; ${ }^{3}$ Rheumatology, Clinique St Odilon, Moulins; ${ }^{4}$ Orthoaedics, Clinique du Parc; ${ }^{5}$ Orthoaedics, Clinique St Charles, Lyon; ${ }^{6}$ Orthoaedics, Inst. Chirurgical de la Main et du Membre Supérieur, Villeurbanne; ${ }^{7}$ Rheumatology, Centre hospitalier, Nevers, France

Background: Viscosupplementation is likely effective to alleviate pain and improve function in patients suffering from rhizarthrosis. However no study has been focused on the predictors of efficacy of the treatment.

Objectives: To search predictive factors of success or failure 3 months after a single intra-articular injection of a mannitol-modified hyaluronic acid (HA) viscosupplement, in patients suffering from trapeziometacarpal (TMC) osteoarthritis $(\mathrm{OA})$.

Methods: Patients with symptomatic TMC OA were included in a 3 month prospective multicentre open-label trial. To be included in the study patients must have symptomatic TMC OA, not adequately relieved by analgesics/NSAIDs therapy and/or by the use of a thumb splint. Before treatment all patients must have had plain radiographs with the Kapandji incidences, for the Dell radiological grade assessment. (1 to 4). Primary endpoints were the variation between injection (D0) and day 90 (D90) of the thumb pain measured on 11 point-Likert scale (0 to 10$)$ and the patient's self-assessment of efficacy ( 0 to 3 ). Treatment consisted in a single injection of 0.6 to $1 \mathrm{ml}$ of HANOX-M-XL, a viscosupplement made of a crosslinked $\mathrm{HA}$ of high molecular weight, from biofermentative origin, combined with mannitol. All injections were performed under fluoroscopic or ultrasound guidance. Predictive factors of pain decrease were studied in univariate and multivariate analysis. All statistical tests were carried out two tailed at the $5 \%$ level of significance.

Results: 122 patients $(76 \%$ females, mean age 60, mean disease duration 36 months) were included and $120(98 \%)$ were assessed at 3 months. $23 \%$ of the TMC OA were grade 1 according to Dell classification, $36.8 \%$ grade $2,36.8 \%$ grade $3 \%$ and $3.5 \%$ grade 4 . At D0, the average (SD) pain level was $6.5 \pm 1.6$ without significant difference between Dell groups $(\mathrm{p}=0.21)$. At day 90 , pain decreased from $6.5 \pm 1.6$ to $3.9 \pm 2.5$ (Difference $-2.7 \pm 2.5 ;-42 \% ; p<0.0001$ ) without significant difference depending on the Dell grade $(p=0.055)$, despite a seemingly smaller number of responders in stage 2 patients. The average analgesic consumption decreased in more than one out of two patients. In univariate analysis, the clinical response was significantly worse in patients taking NSAIDs at baseline $(p=0.012)$, but this difference no longer reached the significance threshold in the multivariate analysis. In multivariate analysis no predictor of response was identified. There was no safety issue. All AEs (11\%) were transient increase of pain during or following $\mathrm{HA}$ administration and resolved without sequel within 1 to 7 days. Conclusions: This study, of the largest cohort of patients treated with viscosupplementation in TMC OA, suggests that a single course of HANOX-M-XL injection is effective in relieving pain, without safety concern. Interestingly patients with the more advanced stages of OA seemed to benefit the treatment as well as those with less advanced OA.

Disclosure of Interest: J. DAUVISSAT: None declared, T. Conrozier Consultan for: Labrha SAS, Speakers bureau: Labrha SAS, H. Lellouche: None declared, B. MAILLET: None declared, C. Rizzo: None declared, V. Travers: None declared, V. locquet: None declared, S. Mellac-Ducamp: None declared

DOI: 10.1136/annrheumdis-2018-eular.3317 